\title{
Indian Music Instruments Semantic Knowledge Representation
}

\author{
Sunitha Abburu,Ph.D \\ Director, Dept of Computer Applications \\ Adhiyamaan Collegeof Engineering \\ Hosur, Krishnagiri - 635 109,Tamilnadu,India
}

\author{
G Suresh Babu \\ Department of M.C.A \\ Adhiyamaan Collegeof Engineering \\ Hosur, Krishnagiri - 635 109,Tamilnadu,India
}

\begin{abstract}
Semantic Web is an intelligent incarnation and advancement in World Wide Web to store, annotate, index and retrieve information by providing knowledge representation, classification, and categorization, a common understanding among the group of people and structuring the information in machine processable format. To structure the information in machine processable semantic models Semantic Web have introduced the concept of ontology. Ontologies are most widely used in artificial intelligence, semantic web, software engineering, information retrieval, knowledge representation, knowledge sharing, knowledge integration, knowledge reuse, and so on. Music plays a vital role in human life. A vast number of musical instruments are used to produce rhythmic sounds. The rapid growth of technology enables on line music. The instruments used and the terms used to describe the concepts varies from individual groups. To support concept based knowledge representation and semantic retrieval music files music instruments knowledge should be identified and represented. This raises the need for construction of Indian music instruments ontology. This ropes semantic web with more intelligent, capable, relevant, responsive interaction and high performance retrieval system. The Indian music instrument ontology is been constructed and the paper concludes with conclusion and future work.
\end{abstract}

\section{Keywords}

Music Instruemtns, Ontology, Semantic Web

\section{INTRODUCTION}

The Semantic Web is the abstract representation of data on the World Wide Web, based on the RDF standards and other standards to be defined. It is being developed by the W3C, in collaboration with a large number of researchers and industrial partners. The term was coined by Tim BernersLee, the inventor of the World Wide Web and director of the World Wide Web Consortium ("W3C")[1], which oversees the development of proposed Semantic Web standards. He defines the Semantic Web as "a web of data that can be processed directly and indirectly by machines". World Wide Web (WWW) is the most popular global information sharing and communication system. Information is provided in text, image, audio and video formats over the web by using HTML which is considered to be unconventional in defining and formalizing the meaning of the context. Most of the available information is unstructured and due to this reason it is very difficult to extract concrete information. Although some search engines and screen scrapers are developed but they are not quite efficient and requires excessive manual preprocessing e.g. designing a schema, cleaning raw data, manually classifying documents into taxonomy and manual post processing. To cope with the existing web based problems .i.e., Information filtration, security, confidentiality and augmentation of meaningful contents in mark-up presentation over the web a semantic based solution "Semantic Web" was introduced by Tim Berners Lee. Semantic Web is an intelligent incarnation and advancement in World Wide Web to collect, manipulate and annotate the information by providing categorization, uniform access to resources and structuring the information in machine processable format.

To structure the information in machine processable semantic models Semantic Web have introduced the concept of "Ontology"[2]. Ontology is the collection of interrelated semantic based modeled concepts based on already defined finite sets of terms and concepts used in information integration and knowledge management. Ontology construction is a highly relevant research issue depending on the extraction of information from web and emergence of ontologies. Ontologies are constructed using some ontology supporting languages like RDF, OWL etc. and connected to each other in a decentralized manner to clearly express semantic contents and arrange semantic boundaries to extract concrete information.

Knowledge representation of musical instruments is a complex task. This involves wide range of musical instrument classifications, descriptions and vides range differences in the vocabulary that is used in practice, in different countries, states and in different social and cultural backgrounds. India is vast country with various social and cultural back grounds. Music plays crucial role in Indians life. India is the world's largest producer of films [3]. The cinema medium gained popularity in the country. Around 1,000 films in various languages of India are produced annually [4]. In the 20th century, Indian cinema, along with the Hollywood and Chinese film industries, became a global enterprise[5].At the end of 2010 it was reported that in terms of annual film output, India ranks first, followed by Hollywood and China[6]. Enhanced technology paved the way for upgrading from Music established cinematic norms of delivering product, altering the manner in which content reached the target audience, as per regional tastes. Indian cinema found markets in over 90 countries where films from India are screened [7]. Indian cinema is a substantial revenue generator accounting for $4-5 \%$ of the net revenues generated by a film in India [8].

The above factors motivate the knowledge representation of Indian musical instruments. Ontologies that define relationships between entities are better than traditional taxonomies at providing meaningful answers to queries. This will support semantic based knowledge representation and 
retrieval. Intelligent machine readable knowledge representation could be achieved through construction of Indian music instrument ontology. Ontologies that define relationships between entities are better than traditional taxonomies at providing meaningful answers to queries.

The rest of the paper is organized as follows. Section 2 describes the literature survey. Section 3 discusses the knowledge representation of Indian music instrument concepts its properties. Finally, the paper concludes with conclusion and future work.

\section{RELATED WORK}

Semantics means meaning. Meaning is about context, content and relationships. Semantic technology in computer field objective is to educate the system and make the computer more intelligent by giving the knowledge about the domain. Giving meanings enables machines as well as people to understand, share and reason with them at execution time. With semantic technologies, adding, changing and implementing new relationships or interconnecting programs is simple. Semantic technologies are "meaning-centered." They include tools for[9]:

- Auto recognition of topics and concepts,

- Information and meaning extraction, and

- Categorization.

Given a question, semantic technologies can directly search topics, concepts, associations that span a vast number of sources.A formal frame work for dealing with music related information on the semantic web including editorial, cultural and acoustic information is discussed in [10]. This ontology can act as grounding for more domain-specific knowledge representation. The Music Ontology is therefore built on top of ontology able to express temporal information: the Timeline Ontology [11]. This ontology is itself built on top of two concepts defined in OWL-Time [12]: Interval and Instant, respectively representing time intervals and instants. The music production process involves physical events that occur at a certain place and time and that can involve the participation of a number of physical objects both animate and inanimate. Such events include performances, involving some musicians and their instruments. On top of these ontologies a number of concepts, relative to the music creation work flow are defined like composition deals with the creation of a Musical Work, arrangement deals with an arrangement of a Musical-Work and performance denotes a particular Performance.

[13] describes the methodologies in the field of knowledge organization that can assist in the conceptual organization of the domain of plucked string instruments in the field of musicology. The author gives Brazilian culture that musicologists have adopted and the problems faced by the researchers faced due to the diversity of terms used which varies with different social groups their location and historical period. The paper has given the contributions of Taborda, Castro, Souza,Affonso. Taborda brings a number of issues regarding the terminology of plucked string instruments. The term violao is only used in Portuguese language in other languages the term guitar derived from Greek is used. Guitare in French gitarre in German ,chitarra in Italian and Guitarra in Spanish. The term viola in Portuguese is used for two types of distinct chordophones -plucked and bowed instruements. Souza raises the question that is it the viola that is called violao or the violao that is called viola. Affonso chose the
Portuguese translations of the term guitar originally French. All these researchers focused on social and cultural differences in Brazilian culture.

[14] [15] presents preliminary work on musical instruments ontology design, and investigates heterogeneity and limitations in existing instrument classification schemes based on the well known Hornbostel and Sach's classification scheme. Representations are developed using the Ontology Web Language (OWL), and compared terminological and conceptual heterogeneity using SPARQL queries. Sefki represents only a preliminary analysis of musical instrument schemes that is based on the well known Hornbostel and Sach's classification scheme[16].

As per our knowledge not much work has been done in the field of Indian music instruments ontology. This motivates the need for construction of Indian music instruments ontology.

\section{INDIAN MUSIC INSTRUMENT ONTOLOGY}

Semantic technologies gives a new scope to handle data and provide an abstraction high level knowledge that enables machine readable and understandable format and interconnection of data, content, and processes. Semantic technologies can be thought of as a new level of depth that provides far more intelligent, capable, relevant, and responsive interaction than with information technologies alone. Fundamental properties are data knows it meaning of its, meaningful connections between data and computer drawn conclusions .The benefits of the semantics are [17]

- $\quad$ Agility - faster, cheaper, flexible and adaptable.

- Integrated-data connections integrated applications

- Intelligent applications new things are possible.

The Indian Music Instrument classification [18], is the current source of the Indian music instrument classification, definitions etc, which the author considers to be the users knowledge. The main beauty of the ontology is that as the users knowledge in domain is enhanced the knowledge can be added and represented very well in the ontology. This flexibility makes ontology developers to develop the ontology with the known and identified concepts and update the ontology as and when the new concepts in that domain are been identified. Indian musical instruments can be broadly classified into four categories, mainly classical, western and folk. The instruments are further sub-classified into the type based on the science behind the same.

- $\quad$ Plucked string instruments

- $\quad$ Bowed string instruments

- Wind instruments

$\begin{array}{cl}\circ & \text { Flute } \\ \circ & \text { Single reed } \\ \circ & \text { Double reed } \\ \circ & \text { Bagpipes } \\ \circ & \text { Free reed } \\ \circ & \text { Free reed and bellow } \\ \circ & \text { Brass } \\ \circ & \text { Other wind instruments }\end{array}$


- Percussion instruments

○ Membranophones

- Hand drums

- Hand frame drums

- $\quad$ Stick and hand drums

- $\quad$ Stick drums

○ Chord percussion

○ Idiophones

- Melodic

- Electronic and so on

Each instrument has got its own definition see table1, table 2 and table 3. This knowledge about the instruments and the relationships among them will enhance the system performance by improving the performance of the retrieval system.

Super concepts like Musical Instrument, Wind Instrument. Sub concepts like Flute, Single Reed, Double-reed, and Bagpipe are the sub concepts of Wind Instruments. Two types of object properties have been identified in the domain for music instruments. They are isA, issiblingof. The 'isA' property exists between every sub concept and Super concept. is A is not symmetric. For example all flutes are wind instruments but every wind instrument need not be a Flute. isA is not functional property. For example Flute is a wind instrument and it is also a musical instrument. But wind instrument and musical instruments are not exactly same. 'isA' is a transitive property. For example flute is a wind instrument and wind instrument is a musical instrument. So we conclude that flute is also a musical instrument.

The 'isSiblingOf', exists among all the sub concepts of a concept. All sub classes of a base class are children of a same parent. isSiblingOf is both symmetric and transitive. Example: Flute is a sibling of Single Reed and Single Reed is also a sibling of flute. So isSiblingOf is a Symmetric.

Example: Flute is a sibling of Single Reed and Single Reed is a sibling of Double Reed that implies Flute is a sibling of Double Reed. isSiblingOf is not functional property. Example: Flute is a sibling of Single Reed and Flute is a sibling of Double Reed it does not mean that Single Reed and Double Reed are same. So the property isSiblingOf is not single valued.

Synonyms can be used to add meaningful, human readable names to ontology elements such as classes, properties and individuals. The Annotation property Label in protégé tool, is used to provide multi-lingual names for ontology elements. Label has range of a string. For example Venu has several meanings in different languages they are pullankuzhal in Tamil, in Malayalam, and koLalu in Kannada. It is known as pillana grovi in Telugu. It is called Bansuri in Marathi, Carnatic flute in English. And Venu synonyms are pullankuzhal, koLalu, pillana grovi, Bansuri and Carnatic flute. Eg : If the query is like 'find music clippings or strings which are played by Flute'. The current technologies retrieve all documents which are having the word as 'flute'. Science the system does not have the knowledge about the flute. The retrieval system will not retrieve the documents that have the concepts of Alghoza, bansuri, venu. If the system is trained within the knowledge about the musical instruments, what is flute, what are the sub concepts of flute, what are the synonyms of the flute what kinds of relationships exists among them and so on. The retrieval performance would be good. If the system has been given the knowledge about the musical instruments that Alghoza, bansuri, venu are flutes. Flute in English, venu is the instrument's ancient Sanskrit name. It is also called by various other names in the languages of South India, including pullankuzhal in Tamil and in Malayalam, and koLalu in Kannada. It is known as pillana grovi in Telugu. It is called Bansuri in Marathi. The knowledge representation and utilization at the time of retrieval results in high performance of the retrieval system. This enables the computer processing more reliable and meaningful. If the system has this knowledge about instruments the retrieval system performance can be enhanced see fig 1, 2 and 3.[29] Protégé is been used as an Ontology developing tool to implement Indian Cinema Music Ontology construction. Protégé (http://protoge.stanford.edu) was developed by Mark Musen's group at Stanford University. Protégé, the ontology editor can be used to design classes in flexible style and to organize classes as hierarchy. The knowledge clarified and represented using the class hierarchies as described above into ontology. Since the Indian music instrument ontology is able to represent the knowledge, the hierarchy of the instrument concepts and the relationships among instruments along with the allies. The design and implementation of Indian music instrument ontology will help all musicians and music lovers, music companies and semantic web to improve the performance of the retrieval system.

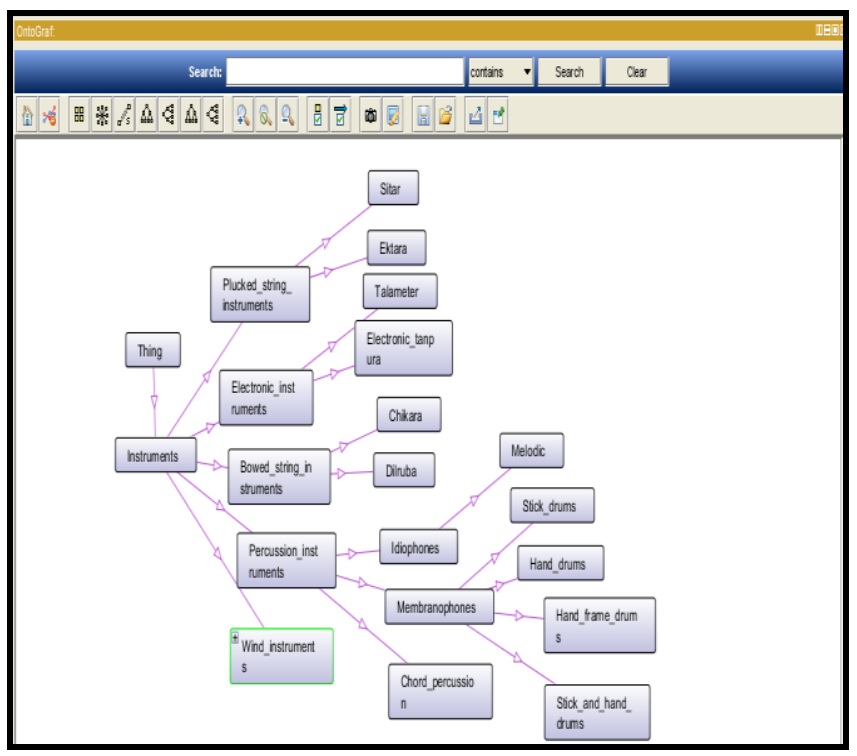

Fig 1: Onto Graph Notation of the String Instruments.

\section{CONCLUSION AND FUTURE WORK}

Ontologies are considered one of the pillars of the Web. Music plays a vital role in human life and the volume of music files is growing rapidly and managing the semantic of the music files is crucial. Due to various social and cultural back grounds in India, has got various types of music instruments. Music instrument ontology defines the knowledge of musical instruments. The knowledge about the instruments classification, the hierarchy and the synonyms trains the system for better performance. Further research could be conducted towards ontology based music instrument annotation and indexing. 
Main Concept

Table 1. Musical Instrument Concept

\begin{tabular}{|c|c|c|c|}
\hline Label & Concept & URI & Definition \\
\hline 1 & $\begin{array}{l}\text { Musical } \\
\text { Instrument }\end{array}$ & $\begin{array}{l}\underline{\text { http://www.music.co.in/instrument.owl\#MusicalIns }} \\
\underline{\text { trument }}\end{array}$ & $\begin{array}{l}\text { A musical instrument is a device created or } \\
\text { adapted for the purpose of making musical }\end{array}$ \\
\hline
\end{tabular}

Table 2. Sub Concepts of MusicalInstrument

\begin{tabular}{|c|c|c|c|}
\hline Label & Concept & URI & Definition \\
\hline 1.1 & $\begin{array}{l}\text { PluckedStringIns } \\
\text { trument }\end{array}$ & $\begin{array}{l}\text { http://www.music.co.in/instrument.owl\#PluckedStr } \\
\text { ingInstrument }\end{array}$ & $\begin{array}{l}\text { Plucked string instruments are a subcategory of } \\
\text { string instruments that are played by plucking } \\
\text { the strings. Plucking is a way of pulling and } \\
\text { releasing the string in such as way as to give it } \\
\text { an impulse that causes the string to vibrate. } \\
\text { Plucking can be done with either a finger or a } \\
\text { plectrum. }\end{array}$ \\
\hline 1.2 & $\begin{array}{l}\text { BowedStringInst } \\
\text { rument }\end{array}$ & $\begin{array}{l}\text { http://www.music.co.in/instrument.owl\#BowedStri } \\
\underline{\text { ngInstrument }}\end{array}$ & $\begin{array}{l}\text { Bowed string instruments are a subcategory of } \\
\text { string instruments that are played by a bow } \\
\text { rubbing the strings. The bow rubbing the string } \\
\text { causes vibration which the instrument emits as } \\
\text { sound. }\end{array}$ \\
\hline 1.3 & WindInstrument & $\begin{array}{l}\underline{\text { http://www.music.co.in/instrument.owl\#WindInstru }} \\
\underline{\text { ment }}\end{array}$ & $\begin{array}{l}\text { A wind instrument is a musical instrument that } \\
\text { contains some type of resonator (usually a } \\
\text { tube), in which a column of air is set into } \\
\text { vibration by the player blowing into (or over) a } \\
\text { mouthpiece set at the end of the resonator. }\end{array}$ \\
\hline 1.4 & $\begin{array}{l}\text { PercussionInstru } \\
\text { ment }\end{array}$ & $\begin{array}{l}\text { http://www.music.co.in/instrument.owl\#Percussion } \\
\text { Instrument }\end{array}$ & $\begin{array}{l}\text { A percussion instrument is any object which } \\
\text { produces a sound when hit with an implement } \\
\text { or when it is shaken, rubbed, scraped, or } \\
\text { otherwise acted upon in a way that sets the } \\
\text { object into vibration. The term usually applies } \\
\text { to an object used in a rhythmic context or with } \\
\text { musical intent. }\end{array}$ \\
\hline 1.5 & $\begin{array}{l}\text { ElectronicInstru } \\
\text { ment }\end{array}$ & $\begin{array}{l}\underline{\text { http://www.music.co.in/instrument.owl\#ElectronicI }} \\
\underline{\text { nstrument }}\end{array}$ & $\begin{array}{l}\text { An electronic musical instrument is a musical } \\
\text { instrument that produces its sounds using } \\
\text { electronics. }\end{array}$ \\
\hline
\end{tabular}

Table 3. Sub Concepts of PluckedStringInstrument

\begin{tabular}{|c|c|c|c|}
\hline Label & Concept & URI & Definition \\
\hline 1.1 .1 & BulbulTarang & $\begin{array}{l}\text { http://www.music.co.in/instrument.o } \\
\text { wl\#BulbulTarang }\end{array}$ & $\begin{array}{l}\text { Bulbul tarang, also known as the "banjo", is a common } \\
\text { instrument in India. The name bulbul tarang literally translates } \\
\text { to "waves of nightingales". It is made of a number of strings } \\
\text { passing over what resembles a finger board. However, instead of } \\
\text { directly fingering the keys, they are pressed with a series of keys } \\
\text { like a piano. Sometimes the keys are similar to a piano } \\
\text { keyboard, but more often they resemble typewriter keys. }\end{array}$ \\
\hline 1.1 .2 & Dotar & $\begin{array}{l}\text { http://www.music.co.in/instrument.o } \\
\text { wl\#Dotar }\end{array}$ & $\begin{array}{l}\text { The dotara (or dotar) is a two or four or some times five stringed } \\
\text { musical instrument resembling more to mandolin than a guitar. } \\
\text { It is commonly used in Assam, Bangladesh, West Bengal \& } \\
\text { Bihar, and dates from the } 15 \text { th-16th century when it was } \\
\text { adopted by the ascetic cults of Bauls and Fakirs. }\end{array}$ \\
\hline 1.1.3 & Ektara & $\begin{array}{l}\text { http://www.music.co.in/instrument.o } \\
\text { wl\#Ektara }\end{array}$ & $\begin{array}{l}\text { Ek means "one" and Tar means"string". The Ektar or -as it is } \\
\text { often called - the Gopi Yantra, is a simple instrument that is } \\
\text { mainly used in Bengal as a folk instrument. }\end{array}$ \\
\hline 1.1 .4 & $\begin{array}{l}\text { GetchuVadya } \\
\mathrm{m}\end{array}$ & $\begin{array}{l}\text { http://www.music.co.in/instrument.o } \\
\underline{\text { wl\#GetchuVadyam }}\end{array}$ & $\begin{array}{l}\text { Getchu vadyam, also known as gettuvadyam, gethu vadyam, or } \\
\text { Jhallari, is a very rare instrument. It is a hammered lute, only } 2 \text { - } \\
3 \text { feet long. It is placed in front of the performer and struck with } \\
\text { two small bamboo mallets. }\end{array}$ \\
\hline 1.1.5 & GottuVadyam & $\begin{array}{l}\text { http://www.music.co.in/instrument.o } \\
\text { wl\#GottuVadyam }\end{array}$ & $\begin{array}{l}\text { The Gottuvadhyam also known as the chitravina, is an } \\
\text { instrument played in Southern India. It is usualy used as a solo } \\
\text { instrument in Carnatic sangeet. }\end{array}$ \\
\hline
\end{tabular}




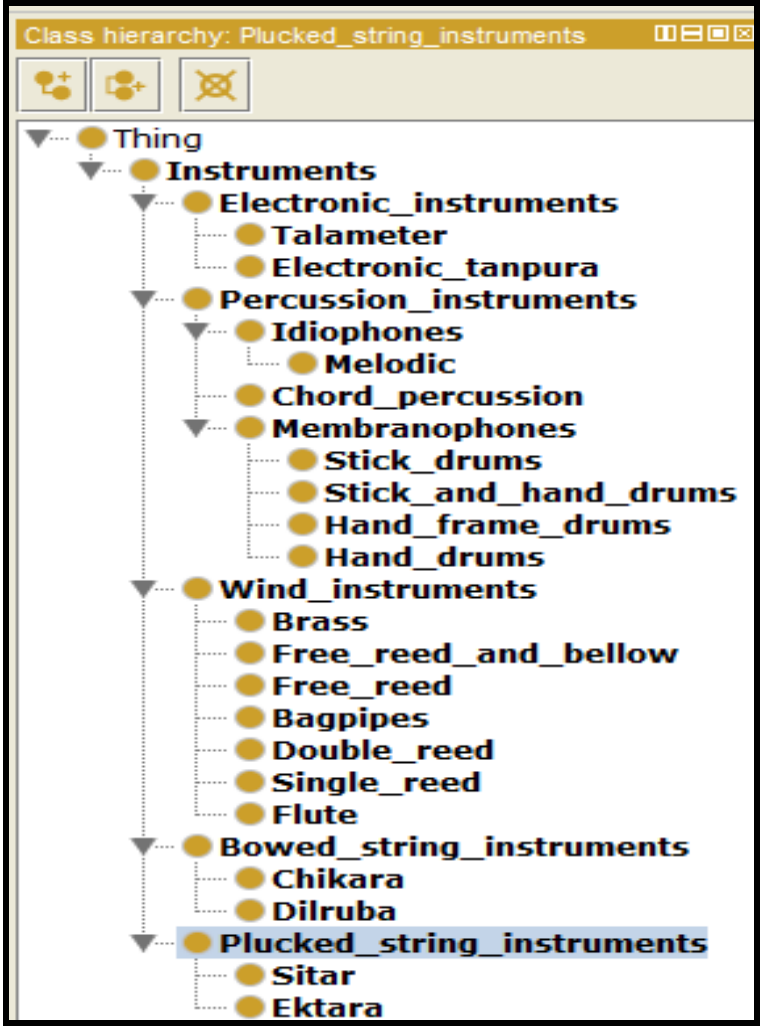

Fig.2 Concepts of Indian Music Instruments

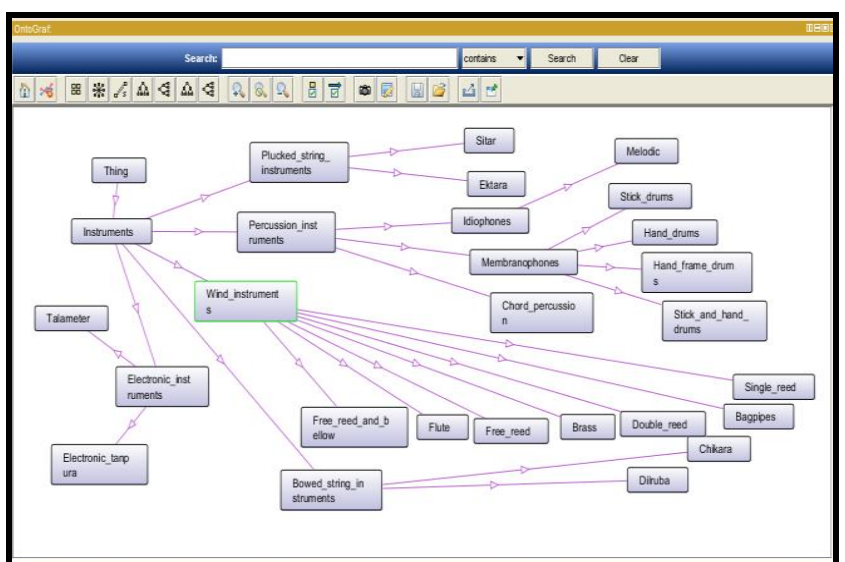

Fig.3 Onto Graph Notation of Indian Music Instruments.

\section{ACKNOWLEDGMENTS}

This work has been partly done in the labs of Adhiyamaan College of Engineering where the author is currently working as a Professor \& Director in the department of Master of Computer Applications. The author would like to express her sincere thanks to Adhiyamaan College of Engineering for their support rendered during implementation of this module.

\section{REFERENCES}

[1] http://en.wikipedia.org/wiki/Semantic_Web.

[2] Heiner, S 2002, "Approximate Information Filtering on the Semantic Web", pp. 114-128, Springer-Verlag M. Jarke et al. (Eds.): KI, LNAI 2479, Berlin Heidelberg Germany.

[3] The Hindu ,"A stalwart on the Malayalam screen". The Hindu. 5 February 1989. Retrieved 29 April 2011.

[4] http://www.sebi.gov.in/dp/gvfilms.pdf

[5] Deccan Chronicle, "Mollywood wants Act to deal with CD piracy". Deccan Chronicle. 26 April 2011. Retrieved 29 April 2011.

[6] The Times Of India, "Chinese film industry races close to Bollywood". The Times Of India, Jan 10, 2011.

[7] Money control.com, "Prime Focus expands India operations ", Money control.com, Indias no 1 Financial portal, Apr 11, 2007.

[8] Kasbekar, Asha , "Pop Culture India!: Media, Arts and Lifestyle” . ABC-CLIO. p. 215. ISBN 978-1-85109-6367, (2006).

[9] http://en.wikipedia.org/wiki/Semantic_technology .

[10] Yves Raimond, Samer Abdallah, Chat Mark Sandler, Frederick Giasson, "The Music Ontology" International Conference on Music Information Retrieval, ISMIR, 2007.

[11] Y. Raimond and S. A. Abdallah, "The timeline ontology," OWL-DL ontology, 2006. [Online]. Available: http://purl.org/NET/c4dm/timeline.owl.

[12] J. R. Hobbs and F. Pan, "Time ontology in owl," Working draft, September 2006. [Online]. Available: http://www.w3.org/TR/owl-time/.

[13] Adriana Onlnto Balleste, " ontology for the Domain of Plucked String Instruments in nineteenth Century Brazil Conceptual Field Definition" IX Congress ISKOSpain, $11^{\text {th }}-13^{\text {th }}$ March 2009.

[14] Sefki Kolozali, Mathieu Barthet, Gy“orgy Fazekas, Mark Sandler ," Knowledge Representation Issues In Musical Instrument Ontology Design ",12th International Society for Music Information Retrieval Conference (ISMIR 2011) ,pp 465-470

[15] Sakurai , The classification of Musical Instruments reconsiderations.

[16] http://en.wikipedia.org/wiki/Hornbostel\%E2\%80\%93Sac hs

[17] Semantic Web: Down to Business, Taxonomy Boot Camp-Washington DC, November 15, 2010.

[18] http://en.wikipedia.org/wiki/Indian_musical_instruments.

[19] Matthew Horridge, Simon Jupp, Georgina Moulton, Alan Rector, Robert Stevens, Chris Wroe. OWL Ontologies using protégé 4 and CO-ODE Tools Edition 1.1. The University of Manchester, October 16,2007. 